\title{
Teoria e eficácia da terapia comportamental dialética na bulimia nervosa e no transtorno da compulsão alimentar periódica
}

\author{
Theory and efficacy of dialectical behavior therapy of \\ bulimia nervosa and binge eating disorder \\ Rui Alexandre Nunes-Costa', Diogo Jorge Pereira do Vale Lamela², Laura Gil-Costa²
}

\section{RESUMO}

Objetivos: Procura-se analisar as atuais evidências empíricas e teóricas sobre o modo de operar nas intervenções comportamentais dialéticas. Procedeu-se igualmente à análise da eficácia dessa terapia no tratamento da bulimia nervosa e no transtorno da compulsão alimentar periódica. Método: Realizou-se uma revisão agregativa da literatura, recorrendo às palavras-chave "dialectical behavior therapy", "bulimia nervosa" e "binge eating disorder" nas bases de dados Psyclnfo e MedLine e em livros da especialidade, sob o critério da atualidade e premência das publicações levantadas. Resultados: A terapia comportamental dialética, inicialmente desenhada para o transtorno de personalidade borderline, tem-se estendido a outros transtornos do eixo I. Sua aplicação às perturbações alimentares sustentase num paradigma dialético com o recurso das estratégias comportamentais e cognitivas.

\section{Palavras-chave}

Terapia comportamental dialética, bulimia nervosa, perturbação de compulsão alimentar periódica.

\section{Keywords}

Dialectical behavior therapy, bulimia nervosa, binge eating disorder. Esse modelo permite aos pacientes uma regulação mais efetiva dos estados afetivos negativos, reduzindo a probabilidade da ocorrência de comportamentos bulímicos e de compulsão alimentar periódica. Conclusão: Embora escasseiem estudos sobre a sua eficácia, os resultados existentes parecem comprovar a eficácia da terapia comportamental dialética nas populações descritas.

\author{
ABSTRACT \\ 1 Universidade do Minho. \\ 2 Universidade do Minho, Serviço de Consulta Psicológica e Desenvolvimento Humano.

Objectives: Current theoretical and empirical evidences on how to operate in dialectical behavioral interventions were examined. The effectiveness of these interventions in the treatment of bulimia nervosa and binge eating disorder were analyzed too. Method: An aggregative literature review was made, using the keywords "dialectical behavior therapy", "bulimia nervosa" and "binge eating disorder", from the database PsycInfo and MedLine and from reference books, selecting the most representative and recent scientific texts about this psychotherapy model. Results: Dialectical behavior therapy, initially designed for borderline personality disorder, has been extended to other disorders of Axis I. Its application to eating disorders is sustained in a dialectic paradigm, using also behavioral and cognitive strategies. This model allows to patients a more effective regulation of negative emotional states, reducing the likelihood of occurrence of bulimic and binge behaviors. Conclusion: Despite the few studies published about his efficacy, the existing results seem to show dialectical behavior therapy effectiveness with populations described. 


\section{INTRODUÇÃO}

A primeira descrição de bulimia nervosa foi elaborada por Russel', em 1979, quando descreveu os casos de 30 pacientes com peso normal, medo intenso de engordar, episódios de compulsão alimentar periódica repetidos e atos purgativos consequentes, nomeadamente o vômito autoinduzido. Nessa primeira descrição, Russel considerava ainda a bulimia nervosa como uma variação da anorexia nervosa. Contudo, em 1991, Fairburn² assumiu esse transtorno de forma autônoma, verificando que apenas de 20\% a 30\% das pacientes bulímicas apresentavam uma história progressiva de anorexia nervosa, em geral de curta duração. Poucos anos mais tarde, e sensível a inúmeros estudos epidemiológicos, a American Psychiatric Association ${ }^{3}$ incorporou, na IV edição do Manual de Diagnóstico e Estatística dos Transtornos Mentais, uma nova seção ao espectro dos transtornos alimentares, a EDNOS (Eating Disorders Not Otherwise Specified), na qual se integra o transtorno da compulsão alimentar periódica.

Como principal característica do transtorno da compulsão alimentar periódica encontra-se a ingestão repetida de grande quantidade de alimentos (binge), acompanhada pela sensação de perda de controle sobre o ato de comer. Associada a essa compulsão alimentar periódica, surge a sensação de mal-estar significativo, distress e, de acordo com estudos empíricos, aumento significativo de peso ${ }^{4,5}$, obesidade e alta comorbilidade com outros transtornos psicológicos ${ }^{6,7}$.

Por seu turno, os indivíduos identificados com bulimia nervosa, apesar de partilharem do critério de compulsão alimentar periódica com o transtorno anterior, apresentam ainda métodos compensatórios recorrentes, com o intuito de prevenir o aumento de peso: uso de laxantes, vômito, recursos diuréticos ou exercício físico exagerado ${ }^{3}$.

Estudos epidemiológicos ${ }^{8-14}$ demonstram que os transtornos alimentares (anorexia nervosa, bulimia nervosa e suas variantes) são quadros psiquiátricos que têm impacto principalmente em adolescentes e jovens adultos do sexo feminino, levando a prejuízos biopsicossociais significativos e com elevadas taxas de mortalidade e morbilidade ${ }^{15}$. Os mesmos estudos ${ }^{8}$ demonstram consistentemente que de $1 \%$ a $3 \%$ de adolescentes do sexo feminino apresentam diagnóstico - ou comportamentos suscetíveis de diagnóstico - de bulimia nervosa; estima-se que $1 \%$ da população geral preencha critérios para um transtorno da compulsão alimentar periódica ${ }^{16}$. Para dar resposta a esses valores e às consequências físicas, psicológicas e sociais desses transtornos, foram desenhadas inúmeras psicoterapias e diversos estudos relativos à sua eficácia. Chen e Linehann", apoiando-se, sobretudo, nos dados que informam que os atuais tratamentos para a bulimia nervosa e para o transtorno da compulsão alimentar periódica são eficazes, a longo prazo, em apenas $50 \%$ dos pacientes com esses transtornos, defendem o desenvolvimento de uma Terapia Comportamental Dialética para esses transtornos. Os mesmos autores insistem ainda no recurso dessa terapia para esse tipo específico de paciente, pelo fato de esses transtornos envolverem dificuldades na regulação afetiva e por esses indivíduos apresentarem escassas competências nessa área, apesar da "aparente competência" em outras áreas da sua vida, dimensões pouco exploradas por outras terapias de relativa eficácia.

A presente revisão da literatura procurou agregar, por um lado, dados relativos aos pressupostos subjacentes à aplicação dessa modalidade terapêutica a pacientes diagnosticados com transtorno da compulsão alimentar periódica e bulimia nervosa e, por outro lado, apresentar dados relativos à sua eficácia terapêutica.

\section{MÉTODO}

Na presente revisão agregativa da literatura, recorreu-se às bases de dados MedLine e Psyclnfo (1980-2007), utilizando os unitermos "dialectical behavior therapy", "bulimia nervosa" e "binge eating disorder". Foram selecionados os artigos mais representativos e recentes sobre o tema. Foi dada especial atenção aos contributos de investigadores de referência internacional no tema. Também foram consultados alguns livros de referência que se justificassem no enquadramento do presente artigo de revisão.

\section{RESULTADOS}

Da pesquisa realizada destaca-se, sobretudo, a escassez de textos obtidos referentes à descrição e à eficácia do tratamento comportamental dialético na bulimia nervosa e no transtorno da compulsão alimentar periódica. O desenvolvimento da terapia comportamental dialética iniciou-se em 1993 ${ }^{18,19}$ para o tratamento do transtorno de personalidade borderline, tendo-se assistido apenas em $1999^{20}$ ao surgimento do primeiro manuscrito dedicado ao estudo da aplicação dessa modalidade terapêutica ao transtorno da compulsão alimentar periódica e, um ano depois, à bulimia nervosa.

\section{A terapia comportamental dialética: do transtorno de personalidade borderline às perturbações do espectro alimentar}

A terapia comportamental dialética (TCD) assenta em princípios cognitivo-comportamentais multimodais e foi originalmente desenhada para o tratamento do transtorno de personalidade borderline ${ }^{18,19}$, sendo a sua aplicação terapêutica conduzida sob a alçada de uma epistemologia dialética 
e as estratégias aplicadas de cariz comportamental. Atualmente, a American Psychiatric Association assume a TCD como o tratamento de regulação afectiva mais eficaz e empiricamente suportado para esse tipo de transtorno ${ }^{21}$.

A lógica dialética nesse transtorno envolve a aceitação dos pacientes nas suas atuais dificuldades - por exemplo, pelo recurso a estratégias como o mindfullness, ao mesmo tempo que faz uso das suas competências para a modificação dos comportamentos desajustados - por meio de um componente didático, análise metódica e interativa das cadeias comportamentais, entre outras estratégias comportamentais.

Apesar de inicialmente ter sido desenvolvida para subgrupos específicos de pacientes com transtorno de personalidade borderline (por exemplo, pacientes com risco acrescido de comportamentos suicidas ou dependentes de substâncias), estudos recentes demonstram a eficácia da TCD para populações diagnosticadas com transtorno de personalidade borderline comórbidas a perturbações alimentares ou com um diagnóstico único de transtorno alimentar em adultos ${ }^{22}$ ou adolescentes 23,24 , ou para outras populações sem diagnóstico no eixo $\|^{25-27}$.

\section{Terapia dialética no tratamento da bulimia nervosa e da compulsão alimentar periódica}

Considerando que central ao transtorno da compulsão alimentar periódica e à bulimia nervosa se encontram os episódios de compulsão alimentar periódica, as terapias efetivas para a bulimia nervosa são frequentemente adaptadas e aplicadas no tratamento da compulsão alimentar periódica. Dessa forma, segue-se um resumo de tarefas e estratégias levadas a cabo por terapeutas comportamentais dialéticos e respectivos pacientes, para o tratamento desses dois transtornos.

Os atuais manuais de intervenção comportamental dialética para esses dois transtornos baseiam-se, primordialmente, no modelo proposto por Linehan ${ }^{18,19}$, em 1993, para o tratamento do transtorno de personalidade borderline. Por exemplo, Telch ${ }^{28}$ apresenta um manual detalhado de TCD para casos específicos de compulsão alimentar periódica, e Salbach-Andrae et al. ${ }^{24}$ seguem as orientações comportamentais dialéticas de Miller et al..$^{25}$ para o tratamento de adolescentes com comportamentos de risco, também essas orientações baseadas no modelo de Linehan, mas especificamente para o tratamento de adolescentes com bulimia e anorexia nervosas.

A TCD para casos de bulimia nervosa ou de compulsão alimentar periódica poderá seguir as modalidades individual ou grupal; o mais comum, num primeiro momento, é iniciar com terapia individual e, depois, seguir com terapia em grupo. O número de sessões poderá oscilar, dependendo das especificidades das populações em tratamento (tipo de transtorno e idade), variando habitualmente entre 16 e 25 semanas ${ }^{24,25}$.

A terapia deverá desenvolver-se, hierarquicamente, de acordo com a severidade da psicopatologia que o paciente apresentar no contexto terapêutico ${ }^{28}$. Assim, a terapia deverá iniciar-se com a diminuição de comportamentos de risco (ideação suicida, por exemplo); depois prestar atenção a comportamentos que interfiram na adesão e no sucesso do tratamento, como a recusa à pesagem ou o não completamento dos registros diários; num terceiro momento reduzir as interferências de comportamentos associados à qualidade de vida, ou seja, a restrição alimentar, o evitamento de eventos e lugares associados à alimentação; e, finalmente, capacitar os pacientes com um conjunto de estratégias comportamentais que lhes permitam regular de forma efetiva os efeitos negativos. Nessa fase existe um conjunto de módulos-chave pelos quais os terapeutas e investigadores dessa corrente se regem, nomeadamente: Orientação para o modelo da TCD para o transtorno em causa; Treino de tolerância ao distress; Treino de competências mindfulness; Treino de competências de regulação emocional; Treino de competências de efetividade interpessoal e, especificamente para perturbações do espectro alimentar ${ }^{24}$, módulos didáticos associados à alimentação e à imagem corporal como, por exemplo, o Acordo entre a alimentação e a imagem corporal.

\section{Orientação para o modelo da TCD para o transtorno em causa}

A componente didática deverá informar os pacientes sobre o papel das emoções na regulação dos frequentes binge. Nesse momento deverá proceder-se também à análise da cadeia comportamental que subjaz a cada binge, permitindo uma análise profunda de um comportamento alimentar problemático. Deverá completar-se essa análise com estratégias de resolução de problemas, com a identificação das competências que o paciente possui e que colaboraram nessa mudança comportamental e das competências que deverá desenvolver ${ }^{29}$. Essa análise obriga o paciente a identificar os fatores desencadeantes de cada comportamento de ingestão alimentar excessivo, os fatores que o tornaram vulnerável, as emoções, cognições e comportamentos associados.

\section{Treino de tolerância ao distress}

Neste módulo o paciente treina estratégias que lhe permitam tolerar momentos ou situações ansiogênicas e que lhe causem distress significativo e que, por determinado motivo, não as possa evitar ou alterar. São estratégias comuns: o self-soothing, estratégias de distração, relaxamento diafragmático ou a aceitação da realidade ${ }^{19,28}$. Essas estratégias pemitem que, perante um evento desestabilizador, esses indivíduos não necessitem recorrer ao ato purgatório ou à compulsão alimentar periódica como forma de alienação. Esse módulo apresenta relativa utilidade para adolescentes, 
por envolver tarefas práticas e concretas e, sobretudo, se as atividades distrativas ministradas envolverem significativos, familiares ou pares.

\section{Treino de competências de atenção (mindfulness)}

Com base nas práticas espirituais zen, o mindfulness poderá ser considerado o âmago da TCD ${ }^{29}$. Essa estratégia, uma vez praticada em contexto terapêutico e aplicada pelos pacientes na sua realidade contextual, permite-Ihes experienciar emoções sem que eles reajam por meio de comportamentos alimentares desajustados. Essa técnica facilita o acesso a uma wise mind (mente sábia), num equilíbrio entre razão e emoção, permitindo-Ihes vivenciar, observar e descrever uma experiência num determinado momento, num equilíbrio entre a experiência emocional e um raciocínio lógico, enfatizando o que é efetivo, e não sob coação de pensamentos dicotômicos envolvendo julgamento de valores morais.

\section{Treino de competências de regulação emocional}

Por meio deste módulo os pacientes aprendem a identificar e compreender as funções das próprias emoções, a diferenciá-las, a reduzir a vulnerabilidade para expressar e vivenciar apenas emoções intensas, a aumentar a possibilidade de vivenciar eventos de forma mais positiva e a modificar experiências emocionais se assim acharem necessário.

\section{Treino de competências de efetividade interpessoal}

O presente treino recorre a exercícios em que se ensaia e treina um conjunto de cenários prováveis no âmbito de relacionamentos interpessoais e em que se pretende que os pacientes apresentem soluções de desenvolvimento adequadas - a elaboração de um pedido de desculpas, um pedido de permissão para dormir em casa de um colega, entre outros.

Esses dilemas interpessoais são comuns na adolescência e justificam a inclusão desse módulo quando os pacientes se encontram, sobretudo, nessa faixa etária ${ }^{23}$. Ao desenvolver essas capacidades interpessoais, reduz-se a possibilidade de ocorrência de conflitos que promovam e justifiquem o recurso ao binge ou ao ato de purgação alimentar.

\section{Acordo entre a alimentação e a imagem corporal}

Esse módulo, introduzido por Salbach-Andrae et al. ${ }^{24}$ para adolescentes com bulimia e anorexia nervosas, providencia um componente educacional com respeito à alimentação (por exemplo, planificação das refeições, diferenças metabólicas entre indivíduos, efeitos da restrição alimentar, elaboração de um plano dietético equilibrado e diversificado) e aborda os principais mitos dessa população sobre as dietas e o controle de peso. Esse componente se aproxima da formulação transdiagnóstica de Fairburn et al..$^{30}$ para os transtornos alimentares, assumindo que uma distorção da imagem corporal poderá envolver-se no desenvolvimen- to, manutenção e reincidência desses transtornos. Assim, o paciente fica informado sobre o processo de formação da imagem negativa que tem de si e da insatisfação que detém sobre o seu aspecto físico. São realizados exercícios de exposição a imagens corporais, de forma a reduzir a ansiedade e o evitamento à confrontação com imagens do próprio corpo, de resto muito semelhante à intervenção desenhada por Fairburn et al. ${ }^{30}$. O terapeuta deverá refugiar-se na reestruturação cognitiva para confrontar distorções cognitivas sobre a alimentação e imagem corporal, substituindo cognições enviesadas por outras mais funcionais e realistas ${ }^{24}$.

\section{Eficácia da TCD na bulimia nervosa e no transtorno da compulsão alimentar periódica}

Embora a literatura demonstre uma relação significativa entre atos purgativos ou de compulsão alimentar periódica e uma regulação desajustada dos afetos negativos ${ }^{31}$, apenas em 2001 Safer et al. ${ }^{32}$ apresentaram o primeiro estudo controlado que evidencia a eficácia da TCD para a bulimia nervosa. Os resultados demonstram uma redução significativa do número de binge e atos purgativos quando se introduziu a fase 3 do tratamento, com os módulos-chave, comparativamente a participantes em lista de espera.

No que se refere a estudos sobre a eficácia da TCD em populações diagnosticadas com transtorno da compulsão alimentar periódica, Telch et al. ${ }^{22}$ apresentaram em 2000 o primeiro estudo controlado, evidenciando que $89 \%$ dos indivíduos do grupo sujeito às estratégias dialéticas cessaram os comportamentos de compulsão alimentar periódica até quatro semanas após o término da intervenção, em comparação com apenas $12,5 \%$ do grupo-controle. Evidenciaram-se ainda melhoria significativa da imagem corporal e redução da preocupação relativa à alimentação nos participantes da TCD. Estudos follow-up de 3 e 6 meses demonstraram que $67 \%$ e $56 \%$ dos participantes, respectivamente, continuaram a não recorrer a atos de ingestão compulsiva.

Um outro estudo, de Wilfley et al..$^{33}$, comparou a TCD com a Psicoterapia Interpessoal, em formato de terapia de grupo, para participantes com excesso de peso e que praticavam frequentemente a binge. Os resultados não revelaram diferenças significativas entre as duas intervenções quando se avaliou o número de binge no pós-tratamento.

\section{DISCUSSÃO E PRINCIPAIS CONCLUSÕES}

As psicoterapias mais comuns e amplamente desenvolvidas para o tratamento desse tipo de transtorno focam-se, sobretudo, nos seus fatores de manutenção. Por exemplo, a terapia cognitivo-comportamental para a bulimia nervosa desenvolve-se em torno da violação da restrição alimentar, enquanto a psicoterapia interpessoal, para o mesmo transtorno, concentra-se nos problemas interpessoais que esses 
indivíduos apresentam como resultado dos comportamentos alimentares desajustados.

A recente conceituação transdiagnóstica que Fairburn et al..$^{30}$ fazem dos transtornos do espectro alimentar agrega à conceituação cognitivo-comportamental tradicional quatro mediadores da resposta alimentar atípica: o perfeccionismo clínico, ou seja, um sistema de autoavaliação exacerbado relativo à forma corporal que leva esses pacientes a procurar atingir metas de perfeição acima das suas capacidades físicas; a baixa autoestima, que compromete a adesão desses indivíduos ao tratamento; dificuldades interpessoais, que numa visão mais contextual demonstram que esses indivíduos possuem problemas significativos em suas relações interpessoais, os quais reforçam os comportamentos alimentares desajustados; e finalmente a intolerância afetiva. A TCD debruça-se, sobretudo, sobre esse último potencial fator de manutenção de comportamentos de compulsão alimentar periódica, considerando ainda na sua equação os restantes fatores de manutenção identificados por Fairburn et $a .^{30}$. A premissa sobre a qual a TCD se sustenta defende que comportamentos alimentares inapropriados, mais concretamente o binge, funcionam como modeladores de uma intolerância a determinados estados afetivos em indivíduos com estratégias de regulação afetiva pouco adaptativas. Por outras palavras, os fatores de manutenção dos transtornos alimentares - nomeadamente a distorção da imagem corporal, a baixa autoestima, problemas relacionais, níveis exacerbados de perfeccionismo ou problemas na superação de tarefas desenvolvimentais na idade púbere - são mediados pelos efeitos de respostas emocionais e pela incapacidade que esses indivíduos apresentam em lidar com essas mesmas emoções. Assim, o binge ou os comportamentos bulímicos são encarados como resultado de tentativas de alienação primária ou secundária de emoções aversivas que são ativadas por pensamentos relativos à alimentação, à imagem corporal, ao perfeccionismo ou por problemas de relacionamento interpessoal17. A um nível primário, o binge funciona como uma fuga a respostas fisiológicas indesejadas, atenuando o distress e desviando o foco atencional de pensamentos associados ao mal-estar causado pela incapacidade de enfrentar afetos negativos. A longo prazo, esses comportamentos bulímicos e de compulsão alimentar periódica resultam em emoções secundárias desagradáveis e mal toleradas como a vergonha, que promovem novamente atos alimentares desajustados.

Dessa forma, Wiser e Telch ${ }^{20}$, Waller ${ }^{34}$ e, mais recentemente, Chen e Linehann", concluem que a conceituação da TCD, até então desenvolvida para os comportamentos de risco de pacientes borderline, baseada na ineficácia que esses indivíduos apresentam ao gerir afetos com uma valência negativa, poderia ser um modelo explicativo para os comportamentos inapropriados de pacientes diagnosticados com um transtorno alimentar. De fato, a TCD desenha-se com o intuito de aju- dar os pacientes a regularem de forma mais efetiva uma vasta gama de emoções negativas que, até então, introduziam os atos de compulsão alimentar periódica ou de purga ${ }^{23}$.

De fato, os dados empíricos apresentados anteriormente parecem demonstrar a eficácia, pelo menos a curto prazo, da TCD para populações bulímicas e com relatos de compulsão alimentar periódica. Assim, pode-se concluir acerca da validade teórica da formulação clínica sobre a dificuldade da regulação de impulsos negativos atribuída ao surgimento, à manutenção e à reincidência de atos de compulsão alimentar periódica.

Os dados empíricos relatados, contudo, deverão ser lidos com cautela. Analisando os resultados sobre a eficácia da TCD comparada com outras intervenções existentes e o aumento relativo do número de binge em momentos follow-up, compreende-se a necessidade de mais estudos sobre a efetividade da TCD para essas populações e, sobretudo, acerca dos mecanismos de reincidência desses comportamentos alimentares desajustados que as atuais formulações teóricas não contemplam.

\section{REFERÊNCIAS}

1. Russell GFM. Bulimia nervosa: an ominous variant of anorexia nervosa. Psychol Med. 1979;9:429-48.

2. Fairburn CG. The heterogeneity of bulimia nervosa and its implications for treatment. J Psychosom Res. 1991;35(Suppl 1):3-9.

3. American Psychiatric Association. Diagnostic and statistical manual of mental disorders. $4^{\mathrm{a}}$ ed. Washington DC: American Psychiatric Association; 1994.

4. Doyle J, Bryant-Waugh R. Epidemiology. In: Lask B, Bryant-Waugh R, editors. Anorexia nervosa and related eating disorders in childhood and adolescence (2nd ed.). East Sussex, UK: Psychology Press; 2000. p. 41-61.

5. Spitzer RL, Devlin M, Walsh BT, Hasin D, Wing R, Marcos M, et al. Binge-eating disorder: a multisite field trial of diagnostic criteria. Int J Eat Disord. 1992;11:191-203.

6. Eldredge KL, Agras WS. Weight and shape overconcern and emotional eating in binge eating disorder. Int J Eat Disord. 1996;19:73-82.

7. Telch CF, Stice E. Psychiatric comorbidity in a non-clinical sample of women with binge eating disorder. J Consult Clin Psychol. 1998;66:768-76.

8. Fairburn CG, Beglin SJ. Studies of the epidemiology of bulimia nervosa. Am J Psychiatry. 1990;147:401-8

9. Bulik CM, Sullivan PF, Tozzi F, Furberg H, Lichtenstein P, Pedersen NL. Prevalence, heritability, and prospective risk factors for anorexia nervosa. Arch Gen Psychiatry. 2006;63:305-12.

10. Hay P. The epidemiology of eating disorder behaviors: An australian community-based survey. Int J Eat Disord. 1998;23:371-82.

11. Hoek HW, Van Hoeken D. Review of the prevalence and incidence of eating disorders. Int J Eat Disord. 2003;34:383-96.

12. Hoek HW. Review of the epidemiological studies of eating disorders. Int Rev Psychiatry. 1993;5:61-74.

13. Machado PP, Machado B, Gonçalves S, Hoek $H$. The prevalence of eating disorders not otherwise specified. Int J Eat Disord. 2007;40(3):212-7.

14. Wade TD, Bergin JL, Tiggemann M, Bulik CM, Fairburn CG. Prevalence and long-term course of lifetime eating disorders in an adult Australian twin cohort. Aust N Z J Psychiatry. 2006;40:121-8

15. Bruce B, Agras WS. Binge eating in females: A population-based investigation. Int J Eat Disord. 1992;12:365-73. 
16. Striegel-Moore RH, Franko DL. Epidemiology of binge eating disorder. Int J Eat Disord. 2003;34(Suppl 1):S19-29.

17. Chen EY, Linehan MM. Dialectical behavior therapy for eating disorders. In: Freeman A, editor. Encyclopedia of cognitive behavior therapy. New York: Springer; 2005.

18. Linehan MM. Cognitive behavioral therapy of borderline personality disorder. New York: Guilford Press; 1993.

19. Linehan MM. Skills training manual for treating borderline personality disorder. New York: Guilford Press; 1993.

20. Wiser S, Telch CF. Dialectical behavior therapy for binge-eating disorder. J Clin Psychol. 1999;55:755-68.

21. American Psychiatric Association. Treating borderline personality disorder: a quick reference guide. Washington DC: American Psychiatric Association; 2004.

22. Telch CF, Agras WS, Linehan MM. Group dialectical behavior therapy for binge eating disorder: A preliminary uncontrolled trial. Behav Ther. 2000;31:569-82.

23. Safer DL, Lock J, Couturier JL. Dialectical behavioral therapy modified for adolescent binge eating disorder: A case report. Cognitive and behavioral practice. 2007;14:157-67.

24. Salbach-Andrae H, Bohnekamp I, Pfeiffer E, Lehmkuhl U, Miller AL. Dialectical behavior therapy of anorexia and bulimia nervosa among adolescents: a case series. Cognitive and behavioral practice. 2008;15:415-23.

25. Miller AL, Rathus JH, Linehan MM. Dialectical behavior therapy with suicidal adolescents. New York: Guilford Press; 2007.
26. Lynch TR, Morse JQ, Mendelson T, Robins (J. Dialectical behavior therapy for depressed older adults: a randomized pilot study. Am J Geriatr Psychiatry. 2003;11(1):33-45.

27. Rathus JH, Miller AL. Dialectical behavior therapy adapted for suicidal adolescents. Suicide Life Threat Behav. 2002;32(2):146-57.

28. Telch CF. Emotion regulation skills training treatment for binge eating disorder: Therapist manual. Unpublished manuscript; 1997.

29. Feigenbaum J. Dialectical behaviour therapy: An increasing evidence base. Journal of Mental Health. 2007:16(1):51-68.

30. Fairburn CG, Cooper Z, Shafran R. Cognitive behavior therapy for eating disorders: a "transdiagnostic" theory and treatment. Behav Res Ther. 2003;41:509-28.

31. Abreu CN, Cangelli R. Anorexia nervosa e bulimia nervosa: abordagem cognitivo-construtivista de psicoterapia. Rev Psiq Clin. 2004;31(4):177-83.

32. Safer DL, Telch CF, Agras WS. Dialectic behavior therapy for bulimia nervosa. Am J Psychiatry. 2001;158:632-4.

33. Wiffley D, Welch R, Stein R, Spurrell E, Cohen L, Saelens B, et al. A randomized comparison of group cognitive-behavioral therapy and group interpersonal psychotherapy for the treatment of overweight individuals with binge-eating disorder. Arch Gen Psychiatry. 2002;59(7):713-21.

34. Waller G. The psychology of binge eating. In: CG Fairburn, KD Brownell, editors. Eating disorders and obesity: a comprehensive handbiik. $2^{2 a}$ ed. New York: The Guilford Press; 2003. p. 98-102. 